\title{
Pathogen Detection and Microbiome Analysis of Infected Wheat Using a Portable DNA Sequencer
}

\author{
Yiheng Hu, ${ }^{1}$ Gamran S. Green, ${ }^{1}$ Andrew W. Milgate, ${ }^{2}$ Eric A. Stone,,${ }^{1,3}$ John P. Rathjen, ${ }^{1, \dagger}$ \\ and Benjamin Schwessinger ${ }^{1, \dagger}$ \\ ${ }^{1}$ Research School of Biology, The Australian National University, Acton ACT 2601, Australia \\ ${ }^{2}$ Wagga Wagga Agricultural Institute, New South Wales Department of Primary Industries, Wagga Wagga NSW \\ 2650, Australia \\ ${ }^{3}$ ANU-CSIRO Centre for Genomics, Metabolomics and Bioinformatics, Acton ACT 2601, Australia
}

Accepted for publication 19 March 2019

\begin{abstract}
Fungal diseases of plants are responsible for major losses in agriculture, highlighting the need for rapid and accurate identification of plant pathogens. Disease outcomes are often defined not only by the main pathogen but are influenced by diverse microbial communities known as the microbiome at sites of infection. Here we present the first use of whole genome shot-gun sequencing with a portable DNA sequencing device as a method for the detection of fungal pathogens from wheat (Triticum aestivum) in a standard molecular biology laboratory. The data revealed that our method is robust and applicable to the diagnosis of fungal diseases including wheat stripe rust (caused by Puccinia striiformis f. sp. tritici), Septoria tritici blotch (caused by

Zymoseptoria tritici), and yellow leaf spot (caused by Pyrenophora
\end{abstract}

ABSTRACT tritici repentis). We also identified the bacterial genus Pseudomonas co-present with Puccinia and Zymoseptoria but not Pyrenophora infections. One limitation of the method is the overrepresentation of redundant wheat genome sequences from samples. This could be addressed by long-range amplicon-based sequencing approaches in future studies, which specifically target nonhost organisms. Our work outlines a new approach for detection of a broad range of plant pathogens and associated microbes using a portable sequencer in a standard laboratory, providing the basis for future development of an on-site disease monitoring system.

Keywords: agriculture, microbiome, plant pathology
Plant diseases, especially those caused by fungal pathogens, jeopardize global crop biosecurity. Increased global trade, human migration, environmental changes, and the accelerated emergence of virulence have been identified as causes for increasing prevalence

${ }^{\dagger}$ Corresponding authors: B. Schwessinger; benjamin.schwessinger@anu.edu.au, and J. P. Rathjen; john.rathjen@anu.edu.au

Funding: This project was supported by The Hermon Slade Foundation (HSF_17_04_Rathjen), The Australian National University, and Australian Research Council (DP170102902).

Current address of G. S. Green: School of Biotechnology and Biomolecular Sciences, The University of New South Wales, Kensington, NSW2052, Australia.

*The $e$-Xtra logo stands for "electronic extra" and indicates that seven supplementary figures and two supplementary tables are published online.

The author(s) declare no conflict of interest. of diseases in plants and animals (Fisher et al. 2012). To prevent and manage diseases, rapid detection and identification of their causal agents are crucial. This is particularly important for outbreak management during the incursion of virulent new isolates that overcome classical methods of control such as the use of resistant crop varieties (Bhattacharya 2017; Pretorius et al. 2000). Traditional methods for crop disease diagnosis rely largely on the expertise of pathologists whom identify diseases initially by eye in the field. While molecular methods of pathogen detection also exist, these are generally only capable of identifying specific diseases with low amounts of variation (De Boer and López 2011). For example, the enzyme-linked immunosorbent assay (ELISA) have been applied for the detection of tree pathogen Xylella fastidiosa (Sherald and Lei 1991). Recently, other optical sensor-based methods have been developed for the detection, identification, and quantification of plant diseases (Mahlein 2015). This method has been used to help smallholder farmers for the disease and pest control (PlantVillage, https://plantvillage.psu.edu/). Conventional pathology, however, remains the primary means of pathogen identification and harbors certain limitations: (i) It relies heavily on the physical appearance of 
disease symptoms. (ii) It has difficulty detecting pathogens that do not infect aerial parts of the plant. (iii) Coinfections may lead to conflicting visual symptoms. (iv) No method currently exists for the rapid identification of unknown pathogens during outbreaks-a limitation epitomized by the recent, devastating incursion of South American races of wheat blast fungus in Bangladesh (Callaway 2016).

DNA sequencing-based methods have been applied to address limitations in detection and monitoring of environmental microbial communities (Nezhad 2014). Such assays rely largely on amplification and sequencing of small conserved regions called meta barcodes from pathogen genomes, including the internal transcribed spacer region or Elongation Factor 1 alpha $(E F 1 \alpha)$ and Betatubulin genes for fungal species and $16 \mathrm{~S}$ ribosomal RNA sequences for bacteria (Janda and Abbott 2007; Raja et al. 2017). Combining multiple conserved gene loci, known as multilocus sequence typing (Maiden et al. 1998), can discriminate closely related species based on single nucleotide polymorphisms (SNPs) within amplicon sequences. This has been used, for example, to distinguish different closely related species of Candida that pose distinct risks and epidemiological outcomes (Odds and Jacobsen 2008). Amplicon sequencing also allows for characterization of the microbial community, the microbiome, within a sample. The microbiome can impact plant function, for example, on altering flowering time (Panke-Buisse et al. 2015) or promoting colonization of fungal pathogens such as Botrytis cinerea (Ritpitakphong et al. 2016). Furthermore, the microbiome is likely to contain organisms that interact with disease-causing agents and, as some of these organisms have antimicrobial activities against plant pathogens, could potentially be harnessed for biocontrol strategies (Ellis 2017). Restrictive read lengths generated by the Illumina sequencing platform limits sequence-based identification of microbial species. Standard Illumina sequencing only allows for a maximum amplicon length of $500 \mathrm{bp}$ to be sequenced and, as such, only the genetic variation present within $500 \mathrm{bp}$ is available for taxonomic assignment of the organism in question (Benítez-Páez and Sanz 2017). Use of PCR also biases the abundance of individual amplicons, making estimation of population frequencies problematic (Kennedy et al. 2014).

Next-generation DNA sequencing platforms have developed rapidly during the last half-decade, overcoming the read length issue and facilitating the identification of microbial species (Benítez-Páez and Sanz 2017). Multiple single molecule, long-read sequencing technologies are currently in use. Most predominant among these are single molecular real-time (SMRT) sequencing (PacBio) and nanopore sequencing (MinION; Oxford Nanopore Technologies (ONT)). PacBio SMRT sequencing relies on the detection of fluorescent nucleotides incorporated into a single DNA molecule during its synthesis within a nanoscale observation chamber (Ardui et al. 2018). Nanopore sequencing enables direct sequencing of native DNA by measuring voltage changes across an artificial membrane when a single DNA molecule passes through a nanopore embedded in the membrane of a flowcell (Jain et al. 2016). Advantages of nanopore sequencing include its relatively low cost, pocketsize form and the potential for real-time data analysis, while current disadvantages include relatively low per read accuracy of around 90\% (Leggett and Clark 2017). Because the MinION is a mobile technology independent of large sequencing facilities, it has been useful for on-site sample sequencing in extreme environments such as microbial paleo mats in the Antarctic (Johnson et al. 2017). For rapid pathogen identification, studies have shown a turnaround time of $6 \mathrm{~h}$ for bacterial from clinical samples with MinION (Charalampous et al. 2018), and from seconds to up to $4 \mathrm{~h}$ for detecting cassava virus in Africa (Boykin et al. 2018) — both unachievable with previous technologies. For antibiotic resistance, Břinda et al. (2018) reported their identification of known resistant bacterial strain within five minutes from real time MinION data. Moreover, the MinION provides significantly longer sequencing reads $(\sim 10 \mathrm{~kb})$ than other sequencing platforms such as Illumina, allowing for more genetic information per read and thus more precise identification of the organism from which the read is derived (Benítez-Páez and Sanz 2017; BenítezPáez et al. 2016). Although recent studies have shown important advances in identification of bacterial pathogens using nanopore sequencing from clinical samples (Mitsuhashi et al. 2017; Schmidt et al. 2017), little has been done testing this new sequencing platform in terms of plant pathogens detection and microbiome profiling of diseased plants.

Here, we tested nanopore sequencing for disease diagnosis in field-grown wheat plants and explored its potential for microbiome profiling. We designed a whole-genome shot-gun sequencing (WGS) workflow for identification of major pathogen species from field samples and profiling of microbes present in these samples to the species level. As a proof-of-concept, we applied our sequencing workflow to wheat leaves infected with known fungal pathogens sampled from the field and a fungicide treated control. By mapping sequencing reads to databases, we successfully detected all fungal diseases present in the infected samples including stripe rust (caused by Puccinia striiformis f. sp. tritici), Septoria tritici blotch (caused by Zymoseptoria tritici), and yellow leaf spot (caused by Pyrenophora tritici repentis), and found one disease (Septoria nodorum blotch, caused by Parastagonospora nodorum). We also conducted preliminary characterization of the microbiomes associated with those diseases, identifying the bacterial genus Pseudomonas as co-present with Puccinia and Zymoseptoria infections. Our results suggest that portable nanopore sequencing has a considerable potential for adaptation to a broad range of crop disease diagnoses and environmental monitoring applications under field conditions.

\section{MATERIALS AND METHODS}

Sample collection. Wheat cultivar Crusader (Advanta Seeds Pty. Ltd.) seeds were planted in the disease nursery field on May 2016 at the Wagga Wagga Agricultural Institute (New South Wales [NSW], $\left.35^{\circ} 02^{\prime} 24.3^{\prime \prime} \mathrm{S}, 147^{\circ} 19^{\prime} 09.5^{\prime \prime} \mathrm{E}\right)$ with daily irrigation. Wheat straw infected the previous season with Septoria tritici blotch and yellow leaf spot was allowed to stand in the field to promote development of pseudothecia. This infected material was used to manually inoculate plants in the nursery field at the tillering growth stage (4 to 5 weeks post-emergence) while stripe rust infections occurred naturally at jointing stage ( 7 to 9 weeks post-emergence). Healthy or diseased plants were diagnosed by a plant pathologist from the NSW Department of Primary Industries via symptomatology before samples were collected during September 2016. Five different treatment groups were collected into five separate containers. These included four infection types: three single infections (Puccinia striiformis f. sp. tritici, Z. tritici, and Pyrenophora tritici repentis) and one double infection (Puccinia striiformis $\mathrm{f}$. $\mathrm{sp}$. tritici $+Z$. tritici), and a fungicide-treated control. For each treatment group, three separate entire wheat tillers were pooled in one container and shipped to the Rathjen laboratory, Research School of Biology, The Australian National University. Each biological replicate $(n=4)$ consisted of two independent leaf cuts $(\sim 100 \mathrm{mg})$ from each treatment group containing visible disease symptoms or not in case of the control. The two leaf of each biological replicate were transferred into 2-ml Eppendorf tubes containing one $5 \mathrm{~mm}$ metal bead. All tubes were labeled and stored at $-80^{\circ} \mathrm{C}$ prior to DNA extraction. 
DNA extraction. DNA extractions were performed according to $\mathrm{Hu}$ (2016). Briefly, $100 \mathrm{mg}$ of leaf tissue was homogenized and cells were lysed using cetyl trimethylammonium bromide (CTAB, Sigma-Aldrich) buffer (added RNAse T1, Thermo Fisher, 1,000 units per $1750 \mu \mathrm{l}$ ), followed by a phenol/chloroform/isoamyl alcohol (25:24:1, Sigma-Aldrich) extraction to remove protein and lipids. The DNA was precipitated with $700 \mu \mathrm{l}$ of isopropanol, washed with $1 \mathrm{ml}$ of $70 \%$ ethanol, dried for $5 \mathrm{~min}$ at room temperature, and resuspended in $50 \mu \mathrm{l}$ of TE buffer containing $10 \mathrm{mM}$ Tris and $1 \mathrm{mM}$ EDTA at $\mathrm{pH}$ 8. DNA extractions were performed independently for each biological replicate $(n=4)$, with the operator blind to the identity of each sample. After extraction, DNA was purified with one volume of Agencourt AMPure XP beads (Beckman Coulter, Inc.) according to the manufacturer's protocol and stored at $4^{\circ} \mathrm{C}$. Quality and average size of genomic DNA was visualized by gel electrophoresis with a $1 \%$ agarose gel for $1 \mathrm{~h}$ at 100 volts. Representative gel images for replicate 3 and 4 are shown in Supplementary Figure S1. DNA was quantified by NanoDrop and Qubit (Life Technologies) according to the manufacturer's protocol. DNA quality and purity was evaluated as described previously (Schalamun et al. 2018).

Library construction and sequencing. For each biological replicate, one PCR-based barcoded 1D sequencing libraries were constructed using 1D PCR barcoding workflow (SQK-LSK107 and EXP-PBC001, batch number EP01.10.0005) as previously described in $\mathrm{Hu}$ and Schwessinger (2018) with the omission of the DNA shearing step. Briefly, a dA-tailing reaction was performed on $700 \mathrm{ng}$ of genomic DNA from each sample using the NEBNext Ultra II End-repair/dA-tailing kit (New England Biolabs). Repaired DNA was cleaned up using one volume of AMPure XP beads, washed on a magnetic rack using $70 \%$ ethanol, and eluted with $31 \mu \mathrm{l}$ of nuclease-free water. Thirty microliters of eluate was used for barcode adapter ligation via the NEB Blunt/TA Ligase Master Mix (New England Biolabs) and Barcode Adapter Mix (ONT 1D PCR barcoding kit EXP-PBC001), followed by another wash step on one volume of AMPure XP beads. Twenty nanograms of the adapterligated template in $2 \mu \mathrm{l}$ of nuclease free water was added to each barcoding PCR reaction with $50 \mu$ of LongAmp Taq $2 \times$ Master Mix (New England Biolabs), $46 \mu \mathrm{l}$ of water, and $2 \mu \mathrm{l}$ of PCR Barcodes (ONT 1D PCR barcoding kit EXP-PBC001). The PCR was performed as follows: denaturation at $95^{\circ} \mathrm{C}$ for $3 \mathrm{~min}, 15$ cycles of $95^{\circ} \mathrm{C} / 15 \mathrm{~s} ; 62^{\circ} \mathrm{C} / 15 \mathrm{~s} ; 65^{\circ} \mathrm{C} / 150 \mathrm{~s}$, a final extension step at $65^{\circ} \mathrm{C}$ for $5 \mathrm{~min}$. Barcoded DNA was purified using one volume of AMPure XP beads and pooled into $1-\mu \mathrm{g}$ fractions in a total of $45 \mu \mathrm{l}$ to which $5 \mu \mathrm{l}$ of DNA CS control (ONT 1D ligation sequencing kit SQK-LSK107) was added. A further dA-tailing reaction was performed as above followed by purification on with one volume of AMPure XP beads. DNA was eluted in $31 \mu$ of nuclease-free water. Adapter ligation was carried out using $30 \mu \mathrm{l}$ of end-prepped DNA, $20 \mu \mathrm{l}$ of Adapter Mix (ONT 1D ligation sequencing kit SQKLSK107), and $50 \mu \mathrm{l}$ of NEB Blunt/TA Ligase Master Mix. The adapter-ligated DNA library was purified with one volume of AMPure XP beads using the Adapter Beads Binding buffer (ONT 1D ligation sequencing kit SQK-LSK107) for washing and eluted in $15 \mu \mathrm{l}$ of elution buffer (ONT 1D ligation sequencing kit SQK-LSK107).

Sequencing reactions were performed independently for each biological replicate on a MinION flowcell (R9.4, ONT) connected to a MK1B device (ONT) operated by the MinKNOW software (version 1.5.2 and version 1.6.11). Each flowcell was primed with $1 \mathrm{ml}$ of priming buffer comprising $480 \mu \mathrm{l}$ Running Buffer Fuel Mix $(\mathrm{RBF}, \mathrm{ONT})$ and $520 \mu \mathrm{l}$ of nuclease free water. Twelve microliters of amplicon library was added to a loading mix including $35 \mu \mathrm{l}$ of RBF, $25.5 \mu 1$ of Library Loading beads (ONT library loading bead kit EXP-LLB001, batch number EB01.10.0012), and $2.5 \mu$ of water with a final volume of $75 \mu \mathrm{l}$ and then added to the flowcell via the
SpotON sample port. The "NC_48Hr_sequencing_FLOMIN106_SQKLSK107" protocol was executed through MinKNOW after loading the library. The sequencing run was restarted after $12 \mathrm{~h}$ and stopped after $48 \mathrm{~h}$.

Sequencing analysis. Raw fast 5 files were processed via the Albacore 2.0.2 software (ONT) for basecalling, barcode demultiplexing and quality filtering (Phred quality score of $>7$ ) following manufacturer's recommendations. All reads that passed quality filtering for each barcode were treated in parallel as follows: firstly, NanoLyse (De Coster et al. 2018) was used to remove lambda phage control DNA sequences; secondly, barcode and adapter sequences were trimmed from the ends of reads using Porechop (Wick et al. 2018). To identify middle adapter sequences by Porechop, a 95\% threshold was set, and reads with middle adapter sequences were split into two sequences; thirdly, seqtk (https://github.com/lh3/seqtk) was used to convert the processed fastq file into fasta format for further analysis. The two nucleotide BLAST (blastn, Basic Local Alignment Search Tool, Altschul et al. 1990) searches were performed using the fasta files for each barcode as queries: first, against a custom genome reference database that contained the wheat reference genome (Clavijo et al. 2017), and the genome sequence of three fungal species used in our study: Puccinia striiformis f. sp. tritici: Schwessinger et al. (2018), accessed 09/2017; Z. tritici: Goodwin et al. (2011), version 2, accessed 09/ 2017; Pyrenophora tritici-repentis: Manning et al. (2013), unmasked assembly, accessed 09/2017. The genome of a fourth species suggested to be absent from the studied wheat growing region, Parastagonospora nordorum, as a negative control (Hane et al. (2007), accessed 09/2017. Individual reads were assigned to have originated from a specific DNA sequence based on their best blastn hit (e-value of <0.01) (Zhang et al. 2000). Reads that did not hit the custom-made database were captured using the filterbyname.sh script from the bbmap script package (https:// sourceforge.net/projects/bbmap/). These reads were used as queries to search against the National Center for Biotechnology Information (NCBI) nucleotide database (ftp://ftp.ncbi.nlm.nih.gov/blast/db/, downloaded 09/2017) using blastn and the same settings and species assignment criteria mentioned above. Two Python scripts (QC_and_BLAST.py and creating_final_dataframe.py) that combine all steps from quality control to BLAST outputs and construct dataframes containing statistical and information from BLAST searches for each read were deposited at https://github.com/ Yiheng323/Pathogen_Detection_and_Microbiome_scripts to facilitate the reproducibility of our results. All basecalled fastq data for each sequencing run were deposited at NCBI Short Reads Archive (SRA) under accession number PRJNA493553. The sequencing summary files and final dataframes were deposited as .tab files for each replicate at https://doi.org/10.6084/m9.figshare.7138262. The python scripts used for generating Figures 2 to 5 and Supplementary Figures S2 to S7 were also deposited at https:// github.com/Yiheng323/Pathogen_Detection_and_Microbiome_scripts.

\section{RESULTS}

Sequencing showed variations in yield and consistency in barcode classification. We designed a pipeline for identification of three known wheat pathogens from infected field samples using MinION sequencing. We infected three experimental field plots as disease nurseries with their corresponding pathogens (Puccinia striiformis f. sp. tritici for stripe rust disease, Z. tritici for Septoria tritici blotch, and Pyrenophora. tritici-repentis for yellow leaf spot) commonly found in New South Wales, Australia, and conducted sampling during the 2016 to 2017 growing season (September). The sequencing experiment included three steps (Fig. 1): (i) 
Pathologists collected three plants from each disease nursery field, with visual confirmation of disease symptoms from three single infections plus one double infection (Puccinia striiformis f. sp. tritici $+Z$. tritici) and a negative control comprising the same wheat variety treated with commercial fungicides at the 10- to 11-week growth stage (3 months post-germination). Individual plants were pooled by treatment type and shipped to the laboratory on ice for processing. (ii) We extracted DNA from two independent leaf cuts of each treatment type without knowing the identity of each treatment to eliminate potential bias from operators. (iii) We barcoded each sample to enable pooling of all five sample types into one sequencing run. The five wheat leaf samples were barcoded as follows: leaves infected with one of the three fungal pathogens (barcodes 1 to 3); leaves infected with two pathogens (barcode 4); and fungicide-treated control without disease symptoms (barcode 5). We performed steps ii to iii four times independently as biological replicates, with step iii performed using a MinION 1D PCR barcoding workflow (EXP-PBC001 and SQK-LSK107). The raw data of each nanopore sequencing run were converted into fastq sequencing reads using Albacore software (ONT), with a quality (Q) score for each base estimating the likelihood of the identified base being correct using the Phred scale (Ewing et al. 1998; Ewing and Green 1998). Based on read mean Q scores, Albacore grouped reads into pass $(\mathrm{Q}$ score $>7)$ or fail $(\mathrm{Q}$ score $\leq 7)$ based on manufacturer's recommendations. We binned reads based on the barcode sequences identified by Albacore at both ends of each sequencing read. If no barcode sequence could be identified, we named the corresponding read as unclassified. Overall, $\sim 92$ to $95 \%$ of the total sequence length of all four sequencing runs passed quality filtering (Supplementary Table S1). The total sequence length from the four runs varied from 682 to 5,100 megabase pairs (Mbp). The mean read length ranged from 1,380 to $3,054 \mathrm{bp}$. Proportions of each barcode in each run were relatively even, although 23.3 to $25.7 \%$ of the sequences were unclassified in all four runs (Fig. 2).

Sequence-based identification correctly identifies the fungal species causing wheat diseases. We performed blastn searches for all quality-filtered reads against a custom genome reference database. We assigned the origin of each read based on its best blastn hit within each database (e-value <0.01) using a "winner-takes-all" strategy which only considers the best match of each sequencing read. The custom genome reference database contained the wheat genome and four fungal genomes including Puccinia striiformis f. sp. tritici, Z. tritici, Pyrenophora tritici-repentis, and Parastagonospora nodorum. These represent the host plant genome, three fungal species present in infected field samples, and one fungal species (Parastagonospora nordorum) not expected to be present in the sampling area as a negative control. The majority of reads were assigned to wheat sequences, comprising total sequence length of $90.1 \%$ in flowcell $1,80.1 \%$ in flowcell $2,92.7 \%$ in flowcell 3, and 91.4\% in flowcell 4 (Fig. 3). We found that healthy leaf samples (barcode 5) contained no or minor total length percentages of pathogen-related sequences in all four replicates $(<0.01 \%)$ (Fig. 4). This indicates that our analysis method is robust and does not lead to high background signals in healthy wheat samples (barcode 5). Significantly higher proportions of total sequencing length (0.5 to $5.7 \%$ ) from infected samples (barcode 1 to 4 ) were assigned as pathogen genome-derived. In all cases, the pathogen genome identified by the highest total sequence length corresponded to the pathogen causing the disease as identified by symptomatology (Fig. 4). This indicates that we are clearly able to identify the diseasecausing pathogen via our sequencing workflow in cases of single infections. Infections with two pathogens (barcode 4) were identified less clearly. In barcode 4 from all biological replicates, total sequence length percentages assigned to Puccinia striiformis $\mathrm{f}$. sp. tritici were between 0.1 and $0.4 \%$; close to or lower than the proportion assigned to negative control species Parastagonospora nodorum. The total relative sequence length identifying each major pathogen species within each barcode were similar across all replicates, with around $0.6 \%$ of barcode 1 from Puccinia striiformis f. sp. tritici, 1.9 to $5.0 \%$ of barcode 2 from Z. tritici, 2.1 to $5.7 \%$ of barcode 3 from Pyrenophora tritici-repentis, and 0.1 to $0.4 \%$ of barcode 4 from Puccinia striiformis f. sp. tritici, and 0.6 to $1.2 \%$ from $Z$. tritici. Unexpectedly, we also identified Parastagonospora nodorum in samples derived from different treatment groups in all four biological replicates. To ascertain whether Parastagonospora nodorum was truly present, we extracted all 915 reads that were assigned to have originated from the Parastagonospora nodorum genome in all four biological replicates. We used these reads to perform an open-ended blastn search against the entire NCBI nonredundant nucleotide (nt) database. We found that 344 of 915 reads were assigned to genus Parastagonospora, including 339 reads identifying Parastagonospora nodorum SN15 genome (Supplementary Fig. S2). A further 371 reads hit-related
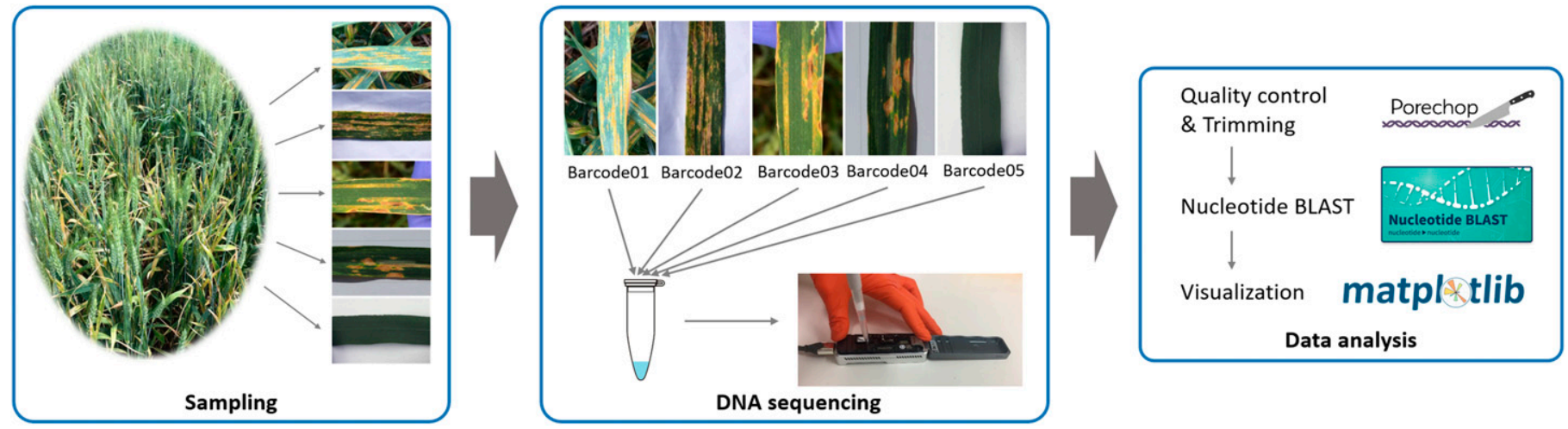

Fig. 1. The Nanopore sequencing workflow for fungal pathogen identification. (i) Sampling: plant pathologists collected wheat leaf samples from disease nursery fields, with visual confirmation of disease symptoms for four infection types (three single fungal infections and one double fungal infection). Fungicide-treated wheat leaves of the same variety and growth stage were collected as a control treatment. (ii) DNA sequencing: to minimize operator bias. DNA was extracted from all five samples without knowledge of the causative agent(s) affecting each sample. We labeled samples with DNA barcodes through a PCR reaction step before sample pooling and 1D library preparations (SQK-LSK107); (iii) Data analysis: we quality-controlled sequences based on Phred quality scores and trimmed adapter sequence using Porechop (Wick et al. 2018). We performed BLAST (Altschul et al. 1990) searches using processed reads and identified the best hits. We summarized the total sequence length of hits to each species using the python matplotlib module. Images used in step iii are from the official websites of each program. 
species, including 226 from the same Pleosporales order: Leptosphaeria spp. (84 reads), Alternaria spp. (81), Pyrenophora spp. (10), Bipolaris spp. (9), Shiraia spp. (9), and other genus. The remaining 200 reads fell above the given e-value threshold (0.01) and failed to be assigned. The difference between both BLAST searches potentially stems from the relatively high error rate of the MinION sequencer and the distinct conserved regions from genomes of closely related species (Jain et al. 2018; Laver et al. 2015). We tested for the presence of Parastagonospora nodorum in one of our four biological replicates using an independent molecular biology approach. We performed a PCR screen in replicate 3 using the PnTox3 gene that is universally found in all Australian Parastagonospora nodorum isolates but not in any other fungal species (Liu et al. 2009). We were able to amplify a specific band corresponding to PnTox3 (Lin et al.
2018, Supplementary Fig. S3) in the Z. tritici treatment group (barcode 2), which displayed the most Parastagonospora nodorum assigned sequencing reads (Fig. 4). The Puccinia striiformis f. sp. tritici treatment group (barcode 1) and the Puccinia striiformis f. sp. tritici $+Z$. tritici treatment group (barcode 4) contained a lesser number of Parastagonospora nodorum-assigned sequencing reads, and these appeared to be below the detection threshold of PCR analysis. These results support the finding that Parastagonospora nodorum is present in our samples from the Wagga Wagga wheatgrowing region despite the disease not being commonly found in the region nor the pathogen being purposefully inoculated in the trial.

Bacterial species associated with fungal disease development. To obtain an overview of the microbiome profile in and on wheat leaves infected by different fungal pathogens, we attempted to

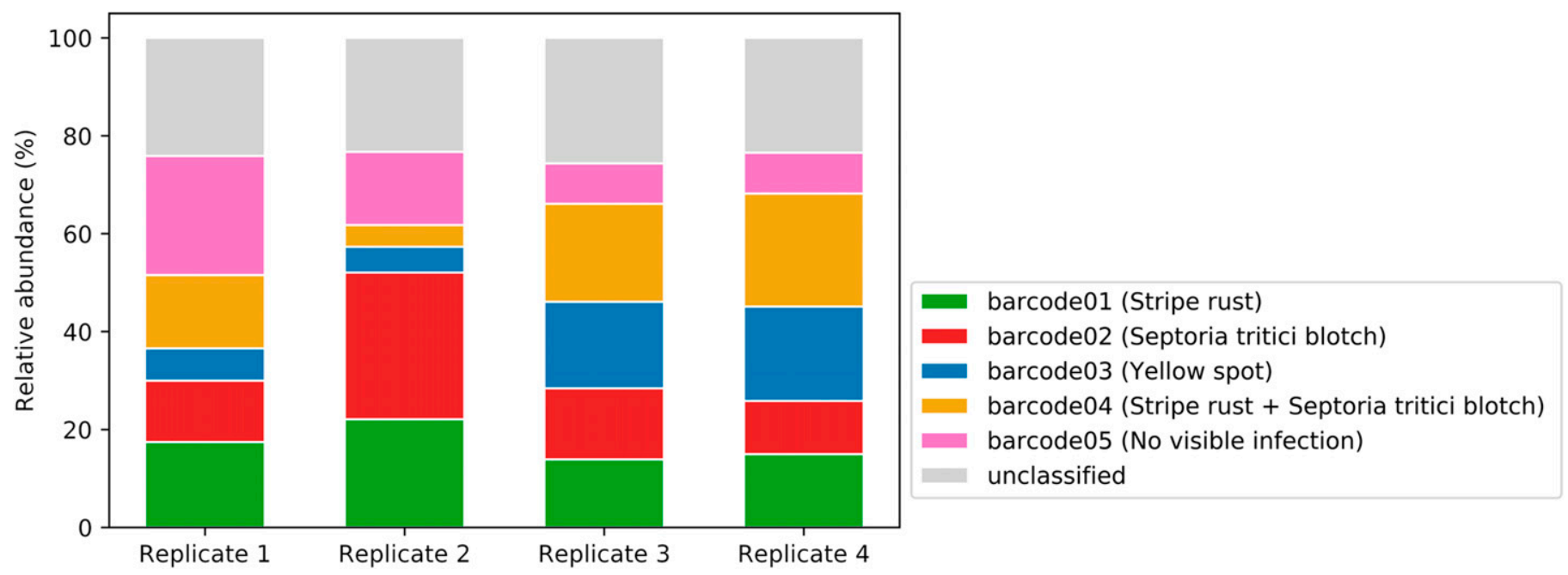

Fig. 2. Uneven proportions of barcode-labeled DNAs across all biological replicates. Columns indicate four biological replicates from five different treatment types. The $y$-axis indicates relative abundance of total sequence lengths labeled with one of five DNA barcodes within each sample set. Each barcode represents one wheat sample. Reads without a detectable barcode were labeled as "unclassified."

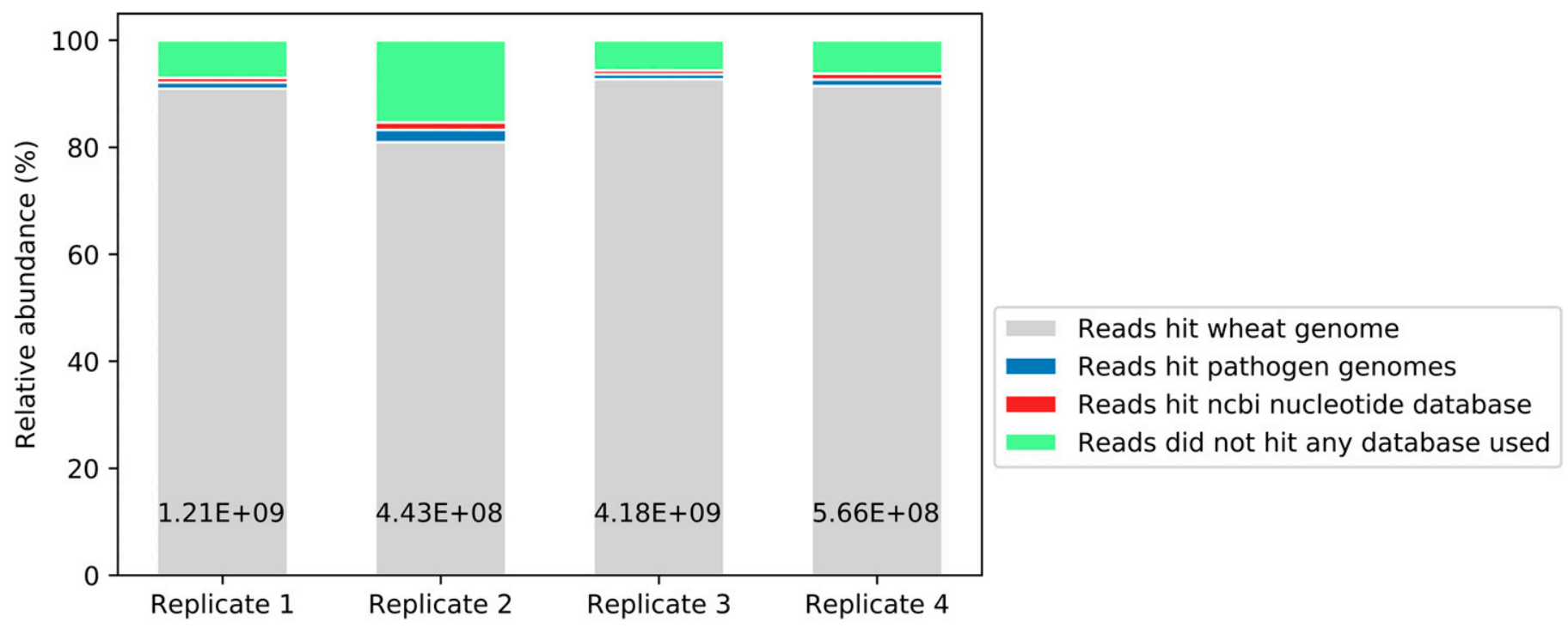

Fig. 3. Wheat is predominantly identified in the whole genome sequencing analysis using BLAST. The graph shows the relative sequence length per species identified using a "winner-takes-all" approach when searching the custom genome reference database. Wheat (gray), fungal pathogen genomes (blue), NCBI nucleotide (nt) database (red), and unmapped (light green) (results and methods sections provide details). The numbers at the bottom of each bar show the total sequence length $(\mathrm{bp})$ of processed reads for each replicate. Replicates 1, 3, and 4 showed similar classification levels, while replicate 2 contained a higher number of unclassified sequences. 
identify other microbial species present on the leaves. We searched the nonredundant NCBI nucleotide database with reads that were not assigned to any of the five reference genomes present in our initial custom genome reference database. We found that the proportion of reads assigned to a genome in the restricted and the ntNCBI databases were high for flowcells 1,3 , and 4 (92.9, 94.3, and $93.8 \%$, respectively), but only $84.6 \%$ for flowcell 2 likely due to technical issues. Overall, from each flowcell, less than $1.4 \%$ of passed reads identified microbiota other than the major pathogens in the custom genome reference database. We pooled the reads from each barcode in all biological replicates, and calculated the total sequence length percentage that identified a specific genus in each sample using NCBI taxonomic identifiers (Fig. 5A). Genus Pseudomonas took up the highest proportion in all diseased samples, ranging from $0.08 \%$ to more than $0.88 \%$, but was nearly absent in healthy wheat (barcode 5, 0.01\%). Genus Alternaria is also found in all samples with a proportion between 0.05 to $0.2 \%$ in diseased samples and $0.005 \%$ in healthy wheat. Uniquely in Puccinia striiformis f. sp. tritici treatment group (barcode 1), bacterial Erwinia comprised $0.09 \%$ of total sequence length as represented by 219 reads and the second highest proportion. In contrast, Erwinia only represented $0.003 \%$ and $0.001 \%$ in Z. tritici treatment group (barcode 2) and fungicide treatment group (barcode 5 ) while being absent in any other treatment group. In treatment groups infected with Z. tritici (barcodes 2 and 4), hits on Z. tritici were still present and may represent sequences in the six $Z$. tritici genomes in the NCBI nucleotide database that are absent from the $Z$. tritici genome we used to generate our initial custom database. At the species level (Fig. 5B), total sequence length percentages identifying Pseudomonas syringae were much more frequent in treatment groups infected with Puccinia striiformis f. sp. tritici (barcode 1, 0.59\%) and/or Z. tritici (barcode 2, 0.33\%). In contrast, the treatment group infected with Pyrenophora tritici-repentis (barcode 3) displayed a much lower relative total sequence length of $0.02 \%$ assigned to Pseudomonas syringae. Relative sequence length assigned to other Pseudomonas species such as Pseudomonas poae followed a similar trend. This finding is consisted with previous reports were Pseudomonas spp. have been descripted to be associated with fungal infections of wheat (Al-Sallami et al. 1997; Mehrabi et al. 2016). At the upper taxonomic classifications, Proteobacteria and Ascomycota were the most abundant bacterial and fungal phyla across all treatment groups (Supplementary Fig. S4) followed by Actinobacteria and Basidiomycota. At the class level (Supplementary Fig. S5), either Gammaproteobacteria or Dothideomycetes were the most abundant. Overall, the microbiome structure of the upper taxonomy resembles that of the lower taxonomy (Supplementary Figs. S6 and S7) as most sequences were assigned to genus Pseudomonas.

\section{DISCUSSION}

We have developed a WGS workflow which accurately identified three fungal pathogens responsible for three major wheat diseases (stripe rust, Septoria tritici blotch, and yellow spot) from field samples. We designed a two-step BLAST process to identify major agents in diseased samples and characterize the associated microbiota. We identified a number of Pseudomonas species present in stripe rust and Septoria tritici blotch-infected wheat leaves, which appear far less abundant in yellow spot or nondiseased leaves.

WGS provides a wealth of information that could be used to accurately identify disease-causing agents and begin to describe microbial community composition in a sample (Brown et al. 2017; Thomas et al. 2012). Without PCR amplification process to potentially introduce bias, WGS data are obtained directly from sample extracts and illustrates features closest to reality dependent only on sampling and DNA extraction strategies. Omitting PCR steps, WGS requires less processing time and proves advantageous
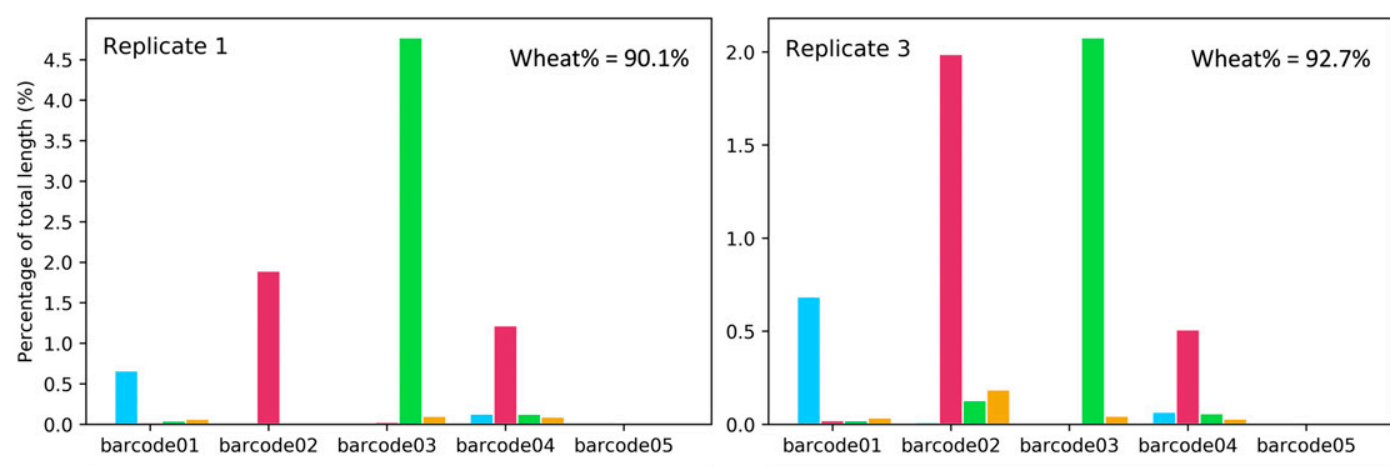

barcode01 barcode02 barcode03 barcode04 barcode05
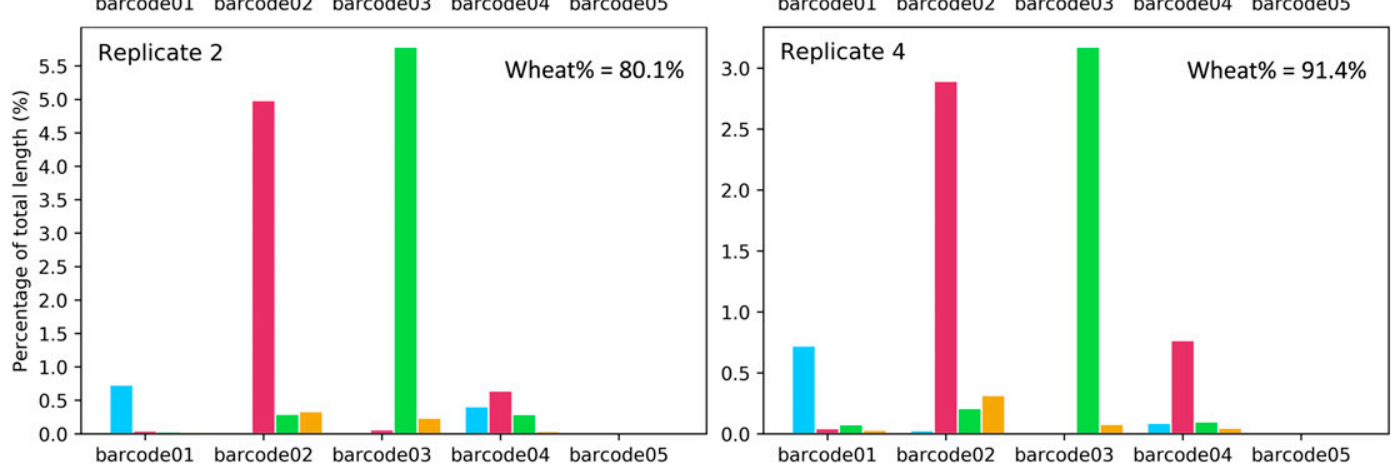

barcode01 barcode02 barcode03 barcode04 barcode05

P.striiformis f. sp tritici
Z.tritici
P.tritici - repentis
P.nodorum

Fig. 4. The total sequence length per pathogen genome per barcode correlated with the phenotypic disease identification. The graphs show the relative sequence length assigned to each pathogen genome using a "winner-takes-all" approach in the BLAST analysis against the custom genome reference database. The proportion of reads hitting the wheat host genome are listed in the top right corner of each graph. Unassigned reads are omitted from the visualization. 
for on-site diagnostic purposes where technical simplicity and fast processing time are prioritized. It has a significant drawback, however, in that most sequences are derived from the host (wheat) genome, representing $90.1 \%$ of total sequence length in flowcell 1,
$80.1 \%$ in flowcell $2,92.7 \%$ in flowcell 3 , and $91.4 \%$ in flowcell 4 . This result is due to our DNA extraction method in which the whole leaf sample was ground to retain all the microbial population in and on the leaf. For the purpose of microbial species identification, host
A

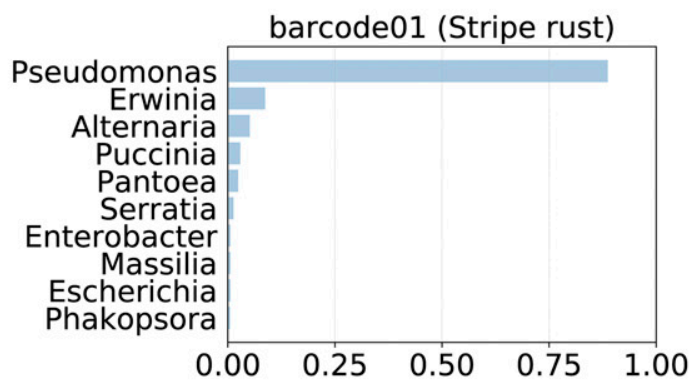

barcode02 (Septoria tritici blotch)

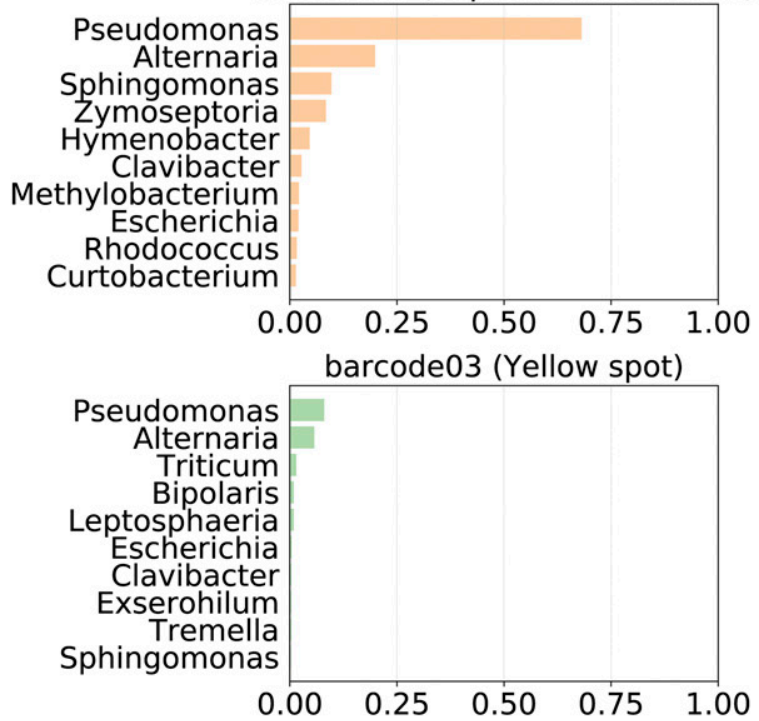

barcode04 (Stripe rust + Septoria tritici blotch)

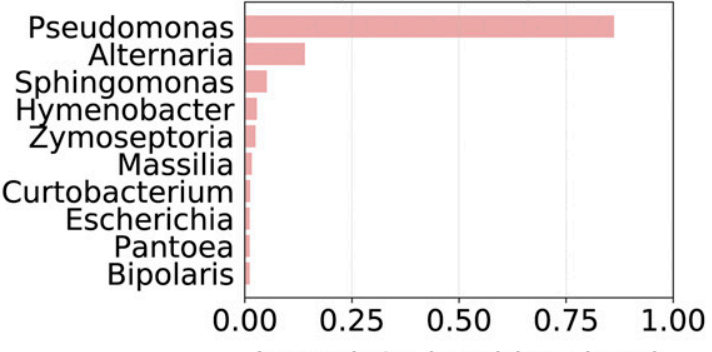

barcode05 (Healthy wheat)

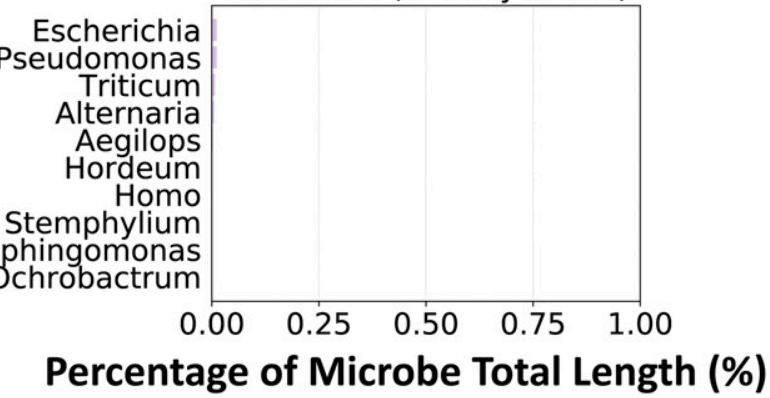

B

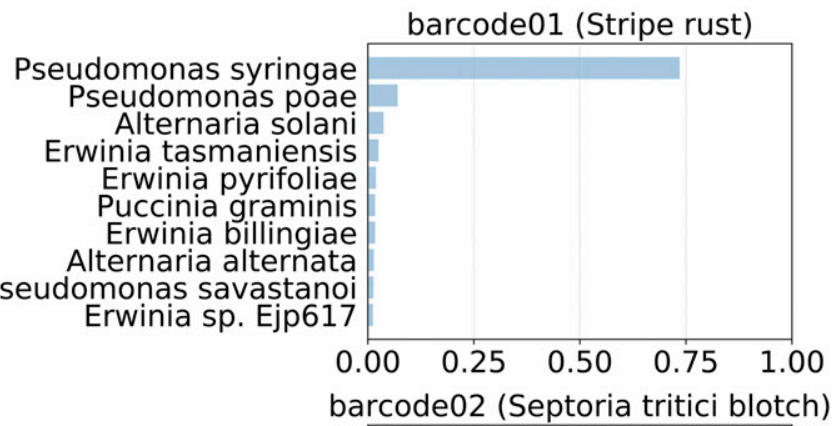
Pseudomonas syringae
Alternaria solani Pseudomonas poae Zymoseptoria tritici Alternaria alternata Pseudomonas viridiflava Sphingomonas taxi Pseudomonas cerasi Hymenobacter sp. PAMC 26554 Escherichia coli

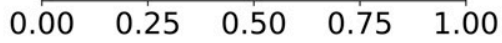

barcode03 (Yellow spot)

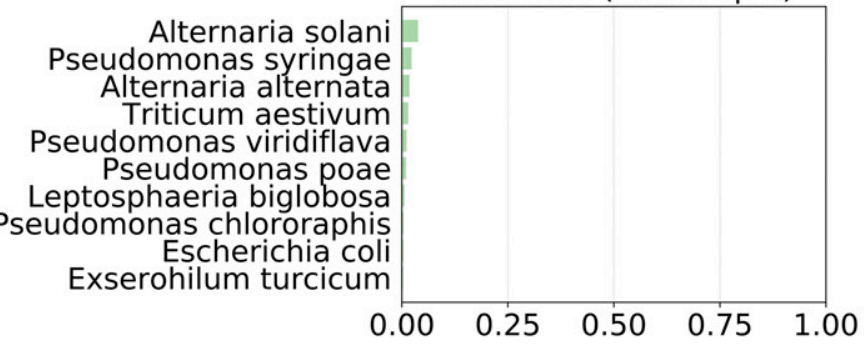

barcode04 (Stripe rust + Septoria tritici blotch)

Pseudomonas syringae

Pseudomonas poae Alternaria solani

Pseudomonas viridiflava

Alternaria alternata

Zymoseptoria tritici

Sphingomonas taxi

Hymenobacter sp. PAMC 26554

Escherichia col

Pantoea agglomerans

$\begin{array}{lllll}0.00 & 0.25 & 0.50 & 0.75 & 1.00\end{array}$

barcode05 (Healthy wheat)

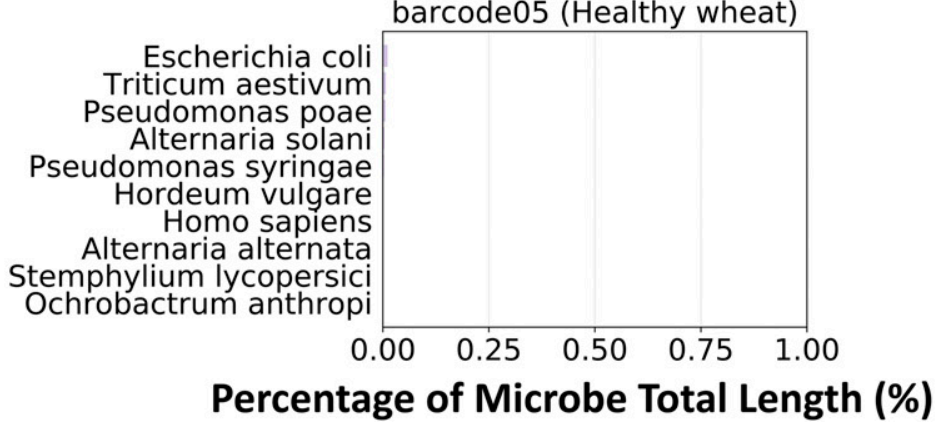

Fig. 5. Fungal infections of wheat increase the microbial population, especially for Puccinia striformis f. sp. tritici and Zymoseptoria tritici infections. Percentage of sequences that did not hit the custom genome reference database but hit the nonredundant NCBI nucleotide database combined from all replicates, and grouped by $\mathbf{A}$, genus or $\mathbf{B}$, species. Different colors represent different treatment types. Reads that were assigned to the restricted database were eliminated, and the top 10 highest classifications were displayed on the $y$-axis for each replicate. Genus Pseudomonas represented the highest proportion across all barcodes containing Puccinia striiformis f. sp. tritici or $Z$. tritici, with Pseudomonas syringae as the highest at the species level. 
sequences are unwanted and can overwhelm community DNA (Sharpton 2014). To enrich microbial sequences, one strategy is to apply intricate molecular methods during DNA extraction such as gradient centrifugation and sonication (Bulgarelli et al. 2012; Lim et al. 2014). As these techniques require sophisticated instruments, this can be a challenge to apply to environmental samples, especially in field conditions. Another strategy to enrich microbiome sequences involves sequencing of microbial metabarcode markers. With the improvement of sequencing length, species-level resolution using long read amplicon sequencing has been achieved in few cases (Benítez-Páez and Sanz 2017; Martijn et al. 2017). Long-read amplicon sequencing seems to be a promising method for species identification of known microbes, as it requires less input DNA than WGS and metabarcode databases contain information from comparatively more species than whole genome databases (Cruaud et al. 2017; Ranjan et al. 2016; Tessler et al. 2017). Amplicon sequencing is also possible in applied cases given the rapid development of remote PCR such as the miniPCR system (Amplyus, Cambridge, U.S.A.) and the Bento Lab platform (Bento Bioworks Ltd., London, United Kingdom). Drawbacks of amplicon strategy include biases related to PCR amplification, which lead to inaccuracy when calculating relative proportions of microbial species.

While many workflows are available for microbiome profiling using metagenomics data, there is no standard method for analyzing long read data. This makes validation of both methods and results crucial (Quince et al. 2017), and mock microbial communities or samples containing known microbial species have been used to validate methods as a proof-of-concept (Nicholls et al. 2018). Verifying the diagnosis of a specific pathogen strain based on SNPs called from MinION reads, Votintseva et al. (2017) first identified pathogens using clinical methods and then generated sequencing data to map this data to the targeted species in real-time. Sequencing reactions were stopped once sufficient SNPs were obtained to confidentially identify the targeted pathogen strain. Similarly, our study used wheat samples inoculated manually by known pathogens and confirmed visually prior to sequencing. The major pathogen identifications from the first BLAST analysis against the custom genome reference database validated the capacity of our workflow for identification of those pathogen species. To validate the usage of a narrow first initial database consisting of suspected pathogens and host, we also performed an open-ended BLAST analysis against the whole nucleotide database with all sequencing reads of replicate 4 (Supplementary Table S2). The overall results of this unrestricted database search are consistent with our targeted analysis approach, both in terms of pathogen identification and microbiome analysis (Figs. 4 and 5). Yet, we observed that some reads were assigned to closely related plant genera such as Aegilops or Hordeum instead of wheat (Triticum). This is likely caused by the presence of highly similar DNA sequences in these related species and the relatively high error rate of nanopore sequencing. To further explore and validate these results different analysis approaches should be performed carefully on the same dataset and compared in detail. Brown et al. (2017) compared the performance of four bioinformatics pipelines on identifying species within different synthetic bacterial communities using MinION. Compared with the composition of a mock community, even the pipeline that resulted in the most similar classification still contains reads classified to species known not to be present. These false positive results are due largely to sequencing errors, which illustrates the need for more stringent filtering of the raw data and improvement of per read accuracy.

Nanopore metagenomics has great potential for quick diagnosis of suddenly emerging crop disease and large-scale disease monitoring at centralized agricultural institutions. As shown from other sequencing studies performed in a more extreme environment
(Boykin et al. 2018; Johnson et al. 2017), all processes including DNA extraction and purification, library preparation and sequencing can be completed within a few hours. A tailored database can be created for each crop species containing the genomes of pathogens known to cause diseases to minimize processing time. For rapidly evolving pathogens such as wheat rusts, the SNP-based classification method described by Votintseva et al. (2017) could be adapted to define rust strains once enough SNPs are obtained. However, before running the sequencer, sampling procedures and tissue processing strategies still need to be carefully curated. In our study, we randomly sampled multiple leaves from different plants belonging to five treatment groups and performed complete sequencing and analysis independently on all four biological replicate sample sets. Consistency in barcode classification and BLAST analysis across all replicates suggested reliability of the workflow. In centralized agricultural institutions, amplicon sequencing can potentially play a key role and, to-date, there have been a few preliminary successes (Benítez-Páez and Sanz 2017; Benítez-Páez et al. 2016; Pomerantz et al. 2018). Given the multiplexing capacity of the 96 native barcoding kit (ONT), many samples can be sequenced in parallel when larger scale sequencing platforms are employed (e.g., GridION, ONT). To achieve this goal, long read databases, sequencing accuracy and access to large-scale sample processing power need to be improved.

In conclusion, our workflow demonstrates the potential of this technology for plant pathogen diagnosis, field applications, and microbiome characterization. A combination of on-site and centralized sequencing approaches would, in future, revolutionize management of agricultural biosecurity and reduce crop loss.

\section{ACKNOWLEDGMENTS}

We thank M. McDonald for providing photos of infected wheat leaves, M. McCaig and T. Goldthorpe for their help during field sampling, P. Solomon for sharing knowledge and PCR primers for Parastagonospora nodorum Tox3 gene, and the editor and two anonymous reviewers for excellent feedback that significantly improved this manuscript.

\section{LITERATURE CITED}

Al-Sallami, F., Karov, S., Vassileva, P., Popova, R., and Vassilev, V. 1997. Pseudomonas syringae pv. atrofaciens associated with fungal black point of wheat (Triticum aestivum) grain. Pages 505-508 in: Pseudomonas syringae Pathovars and Related Pathogens. Springer.

Altschul, S. F., Gish, W., Miller, W., Myers, E. W., and Lipman, D. J. 1990. Basic local alignment search tool. J. Mol. Biol. 215:403-410.

Ardui, S., Ameur, A., Vermeesch, J. R., and Hestand, M. S. 2018. Single molecule real-time (SMRT) sequencing comes of age: Applications and utilities for medical diagnostics. Nucleic Acids Res. 46:2159-2168.

Benítez-Páez, A., Portune, K. J., and Sanz, Y. 2016. Species-level resolution of $16 \mathrm{~S}$ rRNA gene amplicons sequenced through the MinION ${ }^{\mathrm{TM}}$ portable nanopore sequencer. Gigascience 5:4.

Benítez-Páez, A., and Sanz, Y. 2017. Multi-locus and long amplicon sequencing approach to study microbial diversity at species level using the MinION ${ }^{\mathrm{TM}}$ portable nanopore sequencer. Gigascience 6:1-12.

Bhattacharya, S. 2017. Deadly new wheat disease threatens Europe's crops. NATNEWS 542:145

Boykin, L., Ghalab, A., Rossitto De Marchi, B., Savill, A., Wainaina, M. J., Kinene, T., et al. 2018. Real time portable genome sequencing for global food security. F1000 Res. 7:1101.

Břinda, K., Callendrello, A., Cowley, L., Charalampous, T., Lee, R. S., MacFadden, D. R., et al. 2018. Lineage calling can identify antibiotic resistant clones within minutes. bioRxiv 403204.

Brown, B. L., Watson, M., Minot, S. S., Rivera, M. C., and Franklin, R. B. 2017. MinION $^{\mathrm{TM}}$ nanopore sequencing of environmental metagenomes: A synthetic approach. Gigascience 6:1-10. 
Bulgarelli, D., Rott, M., Schlaeppi, K., van Themaat, E. V. L., Ahmadinejad, N., Assenza, F., et al. 2012. Revealing structure and assembly cues for Arabidopsis root-inhabiting bacterial microbiota. Nature 488:91-95.

Callaway, E. 2016. Devastating wheat fungus appears in Asia for first time. NATNEWS 532:421.

Charalampous, T., Richardson, H., Kay, G. L., Baldan, R., Jeanes, C., Rae, D., et al. 2018. Rapid diagnosis of lower respiratory infection using Nanoporebased clinical metagenomics. bioRxiv 387548 .

Clavijo, B. J., Venturini, L., Schudoma, C., Accinelli, G. G., Kaithakottil, G., Wright, J., et al. 2017. An improved assembly and annotation of the allohexaploid wheat genome identifies complete families of agronomic genes and provides genomic evidence for chromosomal translocations. Genome Res. 27:885-896.

Cruaud, P., Rasplus, J.-Y., Rodriguez, L. J., and Cruaud, A. 2017. Highthroughput sequencing of multiple amplicons for barcoding and integrative taxonomy. Sci. Rep. 7:41948.

De Boer, S. H., and López, M. M. 2011. New grower-friendly methods for plant pathogen monitoring. Annu. Rev. Phytopathol. 50:197-218.

De Coster, W., D'Hert, S., Schultz, D. T., Cruts, M., Van Broeckhoven, C., and Berger, B. 2018. NanoPack: Visualizing and processing long-read sequencing data. Bioinformatics 34:2666-2669.

Ellis, J. G. 2017. Can plant microbiome studies lead to effective biocontrol of plant diseases? Mol. Plant-Microbe Interact. 30:190-193.

Ewing, B., and Green, P. 1998. Base-calling of automated sequencer traces using Phred. II. Error Probabilities.Genome Res. 8:186-194.

Ewing, B., Hillier, L., Wendl, M. C., and Green, P. 1998. Base-calling of automated sequencer traces using Phred. I. Accuracy assessment. Genome Res. 8:175-185.

Fisher, M. C., Henk, D. A., Briggs, C. J., Brownstein, J. S., Madoff, L. C., McCraw, S. L., et al. 2012. Emerging fungal threats to animal, plant and ecosystem health. Nature 484:186-194.

Goodwin, S. B., M'Barek, S. B., Dhillon, B., Wittenberg, A. H. J., Crane, C. F., Hane, J. K., et al. 2011. Finished genome of the fungal wheat pathogen Mycosphaerella graminicola reveals dispensome structure, chromosome plasticity, and stealth pathogenesis. PLoS Genet. 7:e1002070.

Hane, J. K., Lowe, R. G. T., Solomon, P. S., Tan, K.-C., Schoch, C. L., Spatafora, J. W., et al. 2007. Dothideomycete-plant interactions illuminated by genome sequencing and EST analysis of the wheat pathogen Stagonospora nodorum. Plant Cell 19:3347-3368.

Hu, Y. 2016. High quality DNA extraction from Fungi_small scale. protocols.io. https://www.protocols.io/view/high-quality-dna-extraction-from-fungismall-scale-exmbfk6

$\mathrm{Hu}$, Y., and Schwessinger, B. 2018. Amplicon sequencing using MinION optimized from 1D native barcoding genomic DNA. protocols.io. https:// www.protocols.io/view/amplicon-sequencing-using-minion-optimizedfrom-1d-mhkc34w

Jain, M., Koren, S., Miga, K. H., Quick, J., Rand, A. C., Sasani, T. A., et al. 2018. Nanopore sequencing and assembly of a human genome with ultra-long reads. Nat. Biotechnol. 36:338-345.

Jain, M., Olsen, H. E., Paten, B., and Akeson, M. 2016. The Oxford Nanopore MinION: Delivery of nanopore sequencing to the genomics community. Genome Biol. 17:239.

Janda, J. M., and Abbott, S. L. 2007. 16S rRNA gene sequencing for bacterial identification in the diagnostic laboratory: Pluses, perils, and pitfalls. J. Clin. Microbiol. 45:2761-2764.

Johnson, S. S., Zaikova, E., Goerlitz, D. S., Bai, Y., and Tighe, S. W. 2017. Realtime DNA sequencing in the Antarctic dry valleys using the Oxford Nanopore Sequencer. J. Biomol. Tech. JBT. 28:2-7.

Kennedy, K., Hall, M. W., Lynch, M. D. J., Moreno-Hagelsieb, G., and Neufeld, J. D. 2014. Evaluating bias of Illumina-based bacterial 16S rRNA gene profiles. Appl. Environ. Microbiol. 80:5717-5722.

Laver, T., Harrison, J., O'Neill, P. A., Moore, K., Farbos, A., Paszkiewicz, K., et al. 2015. Assessing the performance of the Oxford Nanopore Technologies MinION. Biomol Detect. Quantif. 3:1-8.

Leggett, R. M., and Clark, M. D. 2017. A world of opportunities with nanopore sequencing. J. Exp. Bot. 68:5419-5429.

Lim, Y. W., Haynes, M., Furlan, M., Robertson, C. E., Harris, J. K., and Rohwer, F. 2014. Purifying the impure: Sequencing metagenomes and metatranscriptomes from complex animal-associated samples. J. Vis. Exp. 94:e52117.

Lin, S.-Y., Chooi, Y.-H., and Solomon, P. S. 2018. The global regulator of pathogenesis PnCon7 positively regulates Tox3 effector gene expression through direct interaction in the wheat pathogen Parastagonospora nodorum. Mol. Microbiol. 109:78-90.
Liu, Z., Faris, J. D., Oliver, R. P., Tan, K.-C., Solomon, P. S., McDonald, M. C., et al. 2009. SnTox3 acts in effector triggered susceptibility to induce disease on wheat carrying the Snn3 gene. PLoS Pathog 5:e1000581.

Mahlein, A.-K. 2015. Plant disease detection by imaging sensors-Parallels and specific demands for precision agriculture and plant phenotyping. Plant Dis. 100:241-251.

Maiden, M. C. J., Bygraves, J. A., Feil, E., Morelli, G., Russell, J. E., Urwin, R., et al. 1998. Multilocus sequence typing: A portable approach to the identification of clones within populations of pathogenic microorganisms. Proc. Natl. Acad. Sci. USA 95:3140-3145.

Manning, V. A., Pandelova, I., Dhillon, B., Wilhelm, L. J., Goodwin, S. B., Berlin, A. M., et al. 2013. Comparative genomics of a plant-pathogenic fungus, Pyrenophora tritici-repentis, reveals transduplication and the impact of repeat elements on pathogenicity and population divergence. G3 Genes Genomes Genet. 3:41-63.

Martijn, J., Lind, A. E., Spiers, I., Juzokaite, L., Bunikis, I., Pettersson, O. V., et al. 2017. Amplicon sequencing of the 16S-ITS-23S rRNA operon with long-read technology for improved phylogenetic classification of uncultured prokaryotes. bioRxiv 234690.

Mehrabi, Z., McMillan, V. E., Clark, I. M., Canning, G., Hammond-Kosack, K. E., Preston, G., et al. 2016. Pseudomonas spp. diversity is negatively associated with suppression of the wheat take-all pathogen. Sci. Rep. 6:29905.

Mitsuhashi, S., Kryukov, K., Nakagawa, S., Takeuchi, J. S., Shiraishi, Y., Asano, K., et al. 2017. A portable system for rapid bacterial composition analysis using a nanopore-based sequencer and laptop computer. Sci. Rep. 7:5657.

Nezhad, A. S. 2014. Future of portable devices for plant pathogen diagnosis. Lab Chip 14:2887-2904.

Nicholls, S. M., Quick, J. C., Tang, S., and Loman, N. J. 2018. Ultra-deep, longread nanopore sequencing of mock microbial community standards. bioRxiv 487033.

Odds, F. C., and Jacobsen, M. D. 2008. Multilocus sequence typing of pathogenic Candida species. Eukaryot. Cell 7:1075-1084.

Panke-Buisse, K., Poole, A. C., Goodrich, J. K., Ley, R. E., and Kao-Kniffin, J. 2015. Selection on soil microbiomes reveals reproducible impacts on plant function. ISME J. 9:980-989.

Pomerantz, A., Peñafiel, N., Arteaga, A., Bustamante, L., Pichardo, F., Coloma, L. A., et al. 2018. Real-time DNA barcoding in a rainforest using nanopore sequencing: Opportunities for rapid biodiversity assessments and local capacity building. Gigascience 7:goy033.

Pretorius, Z. A., Singh, R. P., Wagoire, W. W., and Payne, T. S. 2000. Detection of virulence to wheat stem rust resistance gene Sr31 in Puccinia graminis $\mathrm{f}$. sp. tritici in Uganda. Plant Dis. 84:203.

Quince, C., Walker, A. W., Simpson, J. T., Loman, N. J., and Segata, N. 2017. Shotgun metagenomics, from sampling to analysis. Nat. Biotechnol. 35:833-844.

Raja, H. A., Miller, A. N., Pearce, C. J., and Oberlies, N. H. 2017. Fungal identification using molecular tools: A primer for the natural products research community. J. Nat. Prod. 80:756-770.

Ranjan, R., Rani, A., Metwally, A., McGee, H. S., and Perkins, D. L. 2016. Analysis of the microbiome: Advantages of whole genome shotgun versus 16S amplicon sequencing. Biochem. Biophys. Res. Commun. 469:967-977.

Ritpitakphong, U., Falquet, L., Vimoltust, A., Berger, A., Métraux, J.-P., and L'Haridon, F. 2016. The microbiome of the leaf surface of Arabidopsis protects against a fungal pathogen. New Phytol. 210:1033-1043.

Schalamun, M., Nagar, R., Kainer, D., Beavan, E., Eccles, D., Rathjen, J. P., et al. 2018. Harnessing the MinION: An example of how to establish longread sequencing in a laboratory using challenging plant tissue from Eucalyptus pauciflora. Mol. Ecol. Resour. 19:77-89.

Schmidt, K., Mwaigwisya, S., Crossman, L. C., Doumith, M., Munroe, D., Pires, C., et al. 2017. Identification of bacterial pathogens and antimicrobial resistance directly from clinical urines by nanopore-based metagenomic sequencing. J. Antimicrob. Chemother. 72:104-114.

Schwessinger, B., Sperschneider, J., Cuddy, W. S., Garnica, D. P., Miller, M. E., Taylor, J. M., et al. 2018. A near-complete haplotype-phased genome of the dikaryotic wheat stripe rust fungus Puccinia striiformis f. sp. tritici reveals high interhaplotype diversity. MBio 9:e02275-17.

Sharpton, T. J. 2014. An introduction to the analysis of shotgun metagenomic data. Front. Plant Sci. 5:209.

Sherald, J. L., and Lei, J. D. 1991. Evaluation of a rapid ELISA test kit for detection of Xylella fastidiosa in landscape trees. Plant Dis. 75:200-203.

Tessler, M., Neumann, J. S., Afshinnekoo, E., Pineda, M., Hersch, R., Velho, L. F. M., et al. 2017. Large-scale differences in microbial biodiversity discovery between $16 \mathrm{~S}$ amplicon and shotgun sequencing. Sci. Rep. 7: 6589 . 
Thomas, T., Gilbert, J., and Meyer, F. 2012. Metagenomics-A guide from sampling to data analysis. Microbiol. Inform. Exp. 2:3.

Votintseva, A. A., Bradley, P., Pankhurst, L., Elias, C. del O., Loose, M., Nilgiriwala, K., et al. 2017. Same-day diagnostic and surveillance data for tuberculosis via whole-genome sequencing of direct respiratory samples. J. Clin. Microbiol. 55:1285-1298.
Wick, R. R., Judd, L. M., and Holt, K. E. 2018. Deepbinner: Demultiplexing barcoded Oxford Nanopore reads with deep convolutional neural networks. PLOS Comput. Biol. 14:e1006583.

Zhang, Z., Schwartz, S., Wagner, L., and Miller, W. 2000. A greedy algorithm for aligning DNA sequences. J. Comput. Biol. J. Comput. Mol. Cell Biol. 7: 203-214. 